\title{
PERIODIC AND LIMIT ORBITS AND THE DEPTH OF THE CENTER FOR PIECEWISE MONOTONE INTERVAL MAPS
}

\author{
ZBIGNIEW NITECKI
}

\begin{abstract}
For a piecewise monotone map of the interval: (a) the nonwandering points outside the closure of the periodic points are isolated in the nonwandering set; (b) the forward orbit of any such point misses all turning points; (c) the depth of the center is at most 2; and (d) all w-limit points belong to the closure of the periodic points.
\end{abstract}

Let $f: I \rightarrow I$ denote a piecewise-monotone map of the interval: $f$ is continuous, and strictly increasing or decreasing on each interval of a finite partition $I=$ $\left[c_{0}, c_{1}\right] \cup \cdots \cup\left[c_{k-1}, c_{k}\right]$. The points $c_{i}(i=1, \ldots, k-1)$ forming the coarsest such partition are the turning points of $f$. Denote the nonwandering set of $f$ by $\Omega$ and the set of periodic points by $P$. In general, $\Omega \neq \bar{P}[\mathrm{~B}],[\mathrm{Y}]$, although generically $\Omega=\bar{P}[\mathbf{Y}]$. However, $\bar{P}$ always contains the recurrent (Poisson stable) orbits [Y], even when $f$ is just continuous [CH]. An analysis of the points in $\Omega-\bar{P}$ is carried out in [Y]; in $[\mathbf{C H}]$ it is shown (for any continuous $f$ ) that $\Omega-\bar{P}$ is nowhere dense in $I$. In this note, we sharpen the analysis in $[\mathrm{Y}]$ to prove the following

THEOREM. If $f: I \rightarrow I$ is piecewise monotone, then

(a) each point of $\Omega-\bar{P}$ is isolated in $\Omega$;

(b) if $x \in \Omega-\bar{P}$, then $f^{n}(x)$ is not a turning point of $f$, for any $n>0$;

(c) $\Omega(f \mid \Omega)=\bar{P}$;

(d) for any $z \in I, \omega(z) \subset \bar{P} \quad(\omega=\omega$-limit set $)$.

Statement (b) for $n=0$ is shown in [Y]. To put statement (c) in perspective, we recall the definition of the Birkhoff center of a dynamical system: let $\boldsymbol{\Omega}^{0}$ be the full phase space ( $I$ in our case); define $\Omega^{i+1}=\Omega\left(f \mid \Omega^{i}\right)$, and for limit ordinals define $\Omega^{i}=\cap\left\{\Omega^{j} \mid j<i\right\}$. For some ordinal $\delta, \Omega^{\delta}=\Omega^{\delta+1}=\cdots$; this set is called the Birkhoff center, and the least such ordinal $\delta$ is called the depth of the center. It has been shown that $\delta \leqslant 2$ for flows on orientable compact surfaces [ST] and $\delta<3$ on nonorientable ones [T], [N1]; on the other hand, $\delta$ is arbitrary for flows on manifolds of higher dimension and on nonorientable open surfaces [N2] (see references there to earlier work of Maìer). Since $\bar{P} \subset \Omega^{i}$ for all $i$, our statement (c) is the equivalent of $\delta \leqslant 2$ for piecewise monotone interval maps.

All the statements of the theorem are easy corollaries of the following technical

Received by the editors October 18, 1979 and, in revised form, December 10, 1979.

AMS (MOS) subject classifications (1970). Primary 54H20; Secondary 58F22. 
Proposition. If $f: I \rightarrow I$ is a piecewise montone map and $x \in \Omega-\bar{P}$, then for some neighborhood of $x, J=J_{-} \cup J_{+}$, where $J_{ \pm}$are closed intervals abutting at $x$, and

(i) $f^{n} J_{+} \cap J=\varnothing$ for all $n>0$;

(ii) $f^{n} J_{-} \cap J \subset J_{+}$for all $n>0$.

I would like to thank Lai-Sang Young and Ethan Coven for helpful conversations concerning these results, and my colleagues at Northwestern University, where I began this work on sabbatical, for their hospitality and support.

Proof of Theorem. To see that the proposition implies (a), (c), and (d) of the theorem, pick $x \in \Omega-\bar{P}$ : we note that (a) int $J_{+}$and int $J_{-}$are both wandering intervals, so $\Omega \cap$ int $J=\{x\}$, (c) since $x$ does not return to $J, \Omega \cap$ int $J$ is wandering for $f \mid \Omega$, and (d) any particular orbit enters $J$ at most twice (once in $J_{-}$, once in $J_{+}$) so that $J$ intersects no $\omega$-limit sets.

To see (b), note that if $f^{n}(x)$ is a turning point for $f$, then $f^{n+1}$ folds some interval $(x-\varepsilon, x+\varepsilon)$, which we can assume is contained in $J$; to every $y \in(x-\varepsilon, x)$ there corresponds $z \in(x, x+\varepsilon)$ such that $f^{n+k}(y)=f^{n+k}(z)$ for all $k>0$. But $y \in J_{-}$iff $z \in J_{+}$; since points of $J_{-}$arbitrarily near $x$ return to $(x-\varepsilon, x+\varepsilon)$ under arbitrarily high iterates of $f$, we have $f^{n+k} J_{+} \cap f^{n+k} J_{-} \cap J \neq \varnothing$ for some large $k$, contradicting (i) of the proposition.

REMARK. The following corollaries of the intermediate value property for the real continuous functions $F_{n}(x)=f^{n}(x)-x$ will be useful in the proof of the proposition:

(1) If $K \subset I$ is a closed interval and either $f^{n}(K) \subset K$ or $f^{n}(K) \supset K$, then $K$ contains a fixed point of $f^{n}$, hence $K \cap P \neq \varnothing$.

(2) If $K \subset I$ is an interval disjoint from $P$ and $f^{n}(y)>y$ (resp. $f^{n}(y)<y$ ) for some $y \in K$, then the same holds for all $y \in K$.

Proof of Proposition. Pick $x \in \Omega-\bar{P}$, and let $J=[x-\varepsilon, x+\varepsilon]$ be a closed neighborhood of $x$, disjoint from $P$ :

(A) $J \cap P=\varnothing$.

Since $x$ is not recurrent (by results mentioned in the introduction), we can adjust $\varepsilon$ so that

(B) $f^{n} x \notin J$ for any $n>0$.

Lemma 1 of [Y] says that

(C) There exist $y_{k} \rightarrow x$ and $n_{k} \rightarrow \infty$ so that $f^{n_{k}} y_{k}=x$.

A subsequence of the $y_{k}$ belongs to one of the half-intervals $[x-\varepsilon, x]$ and $[x, x+\varepsilon]$ : we denote this one by $J_{-}$, and let $J_{+}$be the other half of $J$. We can assume without loss of generality that $J_{-}=[x-\varepsilon, x]$. By adjusting $\varepsilon$, we can assume

(D) $f^{N}(x-\varepsilon)=x$, and $f^{n} y \neq x$ for $n<N$ and $y \in$ int $J_{-}$.

Our first observation is

(E) Lemma. There exists $\delta, 0<\delta<\varepsilon$, so that $f^{n} z \notin(x-\delta, x)$ for any $n>0$ and $z \in J_{+}$. 
Proof of (E). For the moment, pick $0<\delta<\varepsilon$, and suppose for some $z \in J_{+}$ that $f^{\prime} z \in(x-\delta, x)$. Then, since $f^{\prime} z<z$, (A) implies that $f^{\prime} x<x$ and hence by (B), $f^{\prime} x<x-\varepsilon$. Thus,

(E1) $f^{\prime}[x, z] \supset[x-\varepsilon, x-\delta]$.

Now, for $\varepsilon>0$ fixed and $\delta>0$ sufficiently small, one of the points $y_{k}$ in (C) belongs to $(x-\varepsilon, x-\delta)$. Thus, $f^{n_{k}} y_{k}=x>y_{k}$; by (D), $n_{k}>N$, and so $f^{m_{k}}(x-\varepsilon)$ $=f^{n_{k}-N}(x)>x+\varepsilon$ by (A) and (B). Hence,

(E2) $f^{n_{k}}\left[x-\varepsilon, y_{k}\right] \supset[x, x+\varepsilon]$.

But then (E1) and (E2) imply

$$
f^{l+n_{k}}[x, z] \supset f^{n_{k}}[x-\varepsilon, x-\delta] \supset f^{n_{k}}\left[x-\varepsilon, y_{k}\right] \supset[x, x+\varepsilon] \supset[x, z]
$$

contradicting (A).

Note that by remark (1) above, if $f^{n}(z)=x<z$, then $f^{n}(x)<x$, contradicting (E). Thus replacing $\varepsilon$ with $\delta$, we can strengthen (E) to

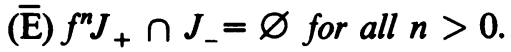

To continue, we invoke piecewise monotonicity. Recall the following notation from [Y]:

$$
\begin{aligned}
\Gamma_{+}(y) & =\operatorname{clos}\left\{f^{n} y \mid n>0\right\}, \\
\Gamma_{-}(y) & =\left\{z \mid \exists y_{k} \rightarrow z, n_{k} \rightarrow \infty \text { with } f^{n_{k}} y_{k}=y\right\} .
\end{aligned}
$$

Since there are finitely many turning points for $f$, we can, by shrinking $J$ further, assume

(F) If $c$ is a turning point of $f$ whose forward and backward orbits both hit $J$, then $x \in \Gamma_{+}(c) \cap \Gamma_{-}(c)$.

By $[Y]$, nonwandering turning points belong to $\bar{P}$. Thus, by more shrinking, we can assume $J$ contains no turning points for $f$.

Next, we show that

(G) If $f^{n} J \cap J$ is nonempty, it is an interval, with one endpoint $x \pm \varepsilon$ and the other either $x$ or $f^{k}(c)$, where $0<k<n$ and $c$ is a turning point as in $(\mathrm{F})$.

Proof of (G). If neither endpoint of $f^{n} J \cap J$ is $x \pm \varepsilon$, then $f^{n} J \subset J$, contradicting (A). On the other hand, $x \pm \varepsilon$ cannot both be endpoints of $f^{n} J$, since $f^{n} J=J$ would also contradict (A). By (E) and remark (1), no image of $x+\varepsilon$ can be interior to $J$; by (B) and (D), the only image of $x-\varepsilon$ interior to $J$ is $x=f^{N}(x-\varepsilon)$. Finally, if an endpoint of $f^{n} J \cap J$ is $f^{n}(y), y \in$ int $J$, then $y$ is a turning point of $f^{n}$, so that some turning point $c$ for $f$ satisfies $c=f^{n-k}(y), f^{n}(y)=f^{k}(c), k>0$, $n-k>0$.

(H) The left endpoint of $f^{n} J \cap J$ is not interior to $J_{-}$.

Proof of (H). If $f^{n} J \cap J=[d, x+\varepsilon]$ and $x-\varepsilon<d<x$, then $d=f^{k}(c)$ for $c$ some turning point, as in (F). Let $(a, b)$ be a maximal interval containing $c$ such that $f^{k}(a, b) \subset$ int $J$. Unless it is an endpoint of the ambient interval $I$, each of the points $a$ and $b$ maps to $x+\varepsilon$; since $f^{k} I \not \subset J$, at least one of the points $a$ and $b$ is not an endpoint of $I$.

Since $x \in \Gamma_{-}(c) \cap(d, x+\varepsilon)$, there exist $y \in f^{n} J \cap J$ and $l$ such that $f^{\prime} y=c$ and hence $f^{l+k} y=d<y$. On the other hand, since $f^{l+k} x \notin J$, some point $z$ between $x$ and $y$ maps under $f^{l}$ to $a$ (or $b$-in any case, not an endpoint of $I$ ) and 
hence $f^{l+k}(z)=x+\varepsilon>z$. But then $f^{l+k}[d, x+\varepsilon] \supset[d, x+\varepsilon]$, contradicting (A).

A further refinement of $(\mathrm{G})$ and $(\mathrm{H})$ is

(I) For each $n>0, f^{n} J \cap J$ (if nonempty) is one of two types: $\left[x-\varepsilon, d_{-}\right]$with $d_{-}<x$, or $\left[d_{+}, x+\varepsilon\right]$ with $d_{+}>x$.

Proof of (I). We know that in the second case $d_{+}>x$ or $d_{+}=x-\varepsilon$. But $d_{+}=x-\varepsilon$ means $f^{n} J J$, contradicting (A). In the first case, if $d_{-}>x$ then $J_{-} \subset f^{n} J \cap J_{-}$; but since $f^{n} J_{+} \cap J_{-}=\varnothing$, we must have $J_{-} \subset f^{n} J_{-}$, again contradicting (A).

Now, consider the finite set of turning points $c_{1}, \ldots, c_{t}$ for $f$ such that $x \in \Gamma_{-}(c)$ $\cap \Gamma_{+}(c)$, and for each $i$, let $d_{i}$ be the first forward image of $c_{i}$ in $J$. Let $d_{0}=x-\varepsilon$, and set $x-\delta=\max \left\{d_{i}<x\right\}$.

(J) LEMMA. $f^{n} J \cap(x-\delta, x)=\varnothing$ for all $n>0$.

Proof of (J). Pick $n$ the least positive integer for which $f^{n} J$ intersects $(x-\delta, x)$. By (I), $f^{n} J \cap J$ must have the form $\left[x-\varepsilon, d_{-}\right]$, with $x-\delta<d_{-}<x$. By (G), $d_{-}=f^{k}\left(c_{i}\right)$ for some $k<n$ and $c_{i}$ one of the turning points above; furthermore, $c_{i} \in f^{n-k} J$. Now, if $d_{-} \neq d_{i}$, then $d_{-}=f^{l} d_{i}$ for some $0<l<k$, and since $d_{i} \in J$, this means $f^{l} J \cap(x-\delta, x) \neq \varnothing$ with $0<l<n$, contradicting the minimality of $n$. But then $d_{-}=d_{i}$, this time contradicting the choice of $x-\delta$. This establishes (J).

The proposition follows easily: by further shrinking $J$, we can assume $J_{-} \subset(x-$ $\delta, x]$ so that $f^{n} J \cap$ int $J_{-}=\varnothing$ (in other words, $f^{n} J \subset J_{+}$) for all $n>0$. On the other hand, $f^{n} J_{+} \cap J_{-}=\varnothing$ for all $n>0$ by $(\overline{\mathrm{E}})$. These two statements give the proposition.

\section{REFERENCES}

[B] L. Block, Diffeomorphisms obtained from endomorphisms, Trans. Amer. Math. Soc. 214 (1975), 403-413.

[CH] E. M. Coven and G. A. Hedlund, $\bar{P}=\bar{R}$ for maps of the interval, Proc. Amer. Math. Soc. 79 (1980), 316-318.

[N1] D. A. Neumann, Central sequences in flows on 2-manifolds of finite genus, Proc. Amer. Math. Soc. 61 (1976), 39-43.

[N2] _ Central sequences in dynamical systems, Amer. J. Math. 100 (1978), 1-18.

[ST] A. J. Schwartz and E. S. Thomas, The depth of the center of 2-manifolds, Proc. Sympos. Pure Math., vol. 14, Amer. Math. Soc., Providence, R. I., 1970, pp. 253-264.

[T] E. S. Thomas, Flows on nonorientable 2-manifolds, J. Differential Equations 7 (1970), 448-453.

[Y] L.-S. Young, A closing lemma on the interval, Invent. Math. 54 (1979), 179-187.

Department of Mathematics, Tufts University, Medford, Massachusetts 02155 